\title{
USOS EDUCATIVO DE LAS TIC Y SU RELACIÓN CON LAS CONCEPCIONES DEL PROFESOR DE HISTORIA SOBRE APRENDER Y ENSEÑAR (1)
}

\section{Marcelo Arancibia Herrera}

Universidad Austral de Chile. Fono: +56-63- 2293178 Correo electrónico: marceloarancibia@uach.cl

(1) Resultados preliminares de la investigación que es parte del proyecto FONDECYT N ${ }^{\circ} 11130316$

\section{Resumen}

La principal finalidad de esta investigación es aportar en el estudio psicoeducativo de las relaciones entre los tipos de concepciones de los profesores de Historia en enseñanza secundaria y las prácticas educativas que llevan a cabo en sus clases con el soporte de las TIC. Teóricamente se asume un posicionamiento respecto de la noción de concepción como conocimiento explícito y situado, asimismo, creemos que las concepciones que los profesores construyen y poseen sobre aprender y enseñar orientan de modo significativo su acción pedagógica.

De este modo el objetivo principal del estudio es establecer patrones de relaciones existentes entre las concepciones de los profesores sobre enseñar y aprender su disciplina con los usos que propone de las TIC en sus prácticas educativas.

La Metodología se basa en un diseño cualitativo, la aplicación de una entrevista semiestructurada a 10 profesores de historia y la filmación en video de 10 secuencias didácticas. La interrelación de los resultados de las entrevistas y las secuencias didácticas filmadas, se hace por medio de una triangulación y del cruce interpretativo de los datos que permite configurar patrones prototípicos entre concepciones y prácticas.

La investigación logró encontrar patrones en el uso de las TIC que hacen los profesores vinculados con sus concepciones sobre aprender y enseñar ya sea de orden constructivista o transmisiva.

Usos educativo de las TIC y su relación con las concepciones del profesor de historia sobre aprender y enseñar 
Palabras clave: tecnologías de la información y la comunicación; concepciones aprender; concepciones enseñar

\section{Antecedentes teóricos}

Generalmente, los autores que han estudiado las concepciones de los profesores sobre la enseñanza o el aprendizaje utilizan modelos construidos de manera previa, a partir de nociones teóricas y conceptuales (Lin y Tsai, 2011). Estos modelos, en una tentativa de clasificación, consideran los paradigmas educativos principales a partir de la construcción de categorías que relacionen los postulados expuestos por cada uno de los enfoques teóricos utilizados (Arancibia, 2011).

Así mismo, suficiente número de investigaciones ya han aportado que las concepciones actúan como filtro o componente decodificador de información que procede de ámbitos externos: desde la investigación, el currículo normativo, las demandas sociales, entre otros (Ruthven, Hennessy y Brindley, 2004). De esta forma las concepciones son un componente fundamental que guía las prácticas de los profesores en las aulas (Kim, Kim, Lee, Spector y DeMeester, 2013; Prestridge, 2012)

Desde una perspectiva epistemológica y de enfoques psicoeducativos (Arancibia, 2011; Gómez y Guerra, 2010), observamos la configuración de una estructura categorial de concepciones sobre aprender y enseñar en tres ejes. La cual dará origen a la matriz preliminar de organización de las concepciones de los profesores en esta investigación, a saber:

A) Externa transmisiva. Las concepciones están sustentadas en una realidad externa objetiva, por tanto su foco está puesto en un "objeto" que se ha de conocer. Es por tanto, una concepción de tipo "reproductiva", que privilegia la entrega de información y un aprendizaje reproductivo por parte de los estudiantes, cercana a posiciones psicoeducativas del procesamiento de la información.

B) Constructiva "individual". En este caso, todo acto de enseñanza está supeditado a las motivaciones y estructuras previas o nivel de maduración cognitiva de los aprendices. Por tanto, un conocimiento que pone acento en el sujeto que conoce, con la salvedad de que esa "subjetividad" se ha de contrastar con la realidad "objetiva" para constituirse efectivamente en conocimiento. Una enseñanza que busca un aprendizaje personal a través de procesos de asimilación y acomodación cercano al 
constructivismo cognitivo, que persigue nuevos equilibrios intrapsicológicos, con ello una construcción individual lo más cercana posible a la realidad externa.

C) Constructiva "social". El conocimiento es una trama o red de relaciones, donde se deconstruyen los roles instituidos. En este caso el foco está puesto en la relación, una concepción de carácter "constructivista" con orientación en el proceso de aprender que tienen como base el constructivismo social, basado en la experiencia humana, como catalizador del aprendizaje, que fomenta prácticas asentadas en la disposición de apoyos didácticos (andamiaje) y la configuración de zonas de desarrollo próximo.

De esta forma, las concepciones se configuran como dispositivo cognitivo fundamental que guía las acciones de los profesores en sus aulas. Por tanto, como hemos adelantado, es vital observar la relación entre las concepciones y el uso de TIC, dado que éstas últimas son identificadas como artefactos culturales vitales en los procesos de cambio educativo actuales.

La investigación sobre la interacción educativa es, al decir de Colomina, Mayordomo y Onrubia (2001), un tema de interés particular de las perspectivas teóricas socio-constructivistas. Pues el foco está puesto en las relaciones y lo efectos de éstas en el aprendizaje, en la lógica de la construcción compartida del conocimiento. De allí que el estudio por la interacción se traslade más que a la acción en si misma al cómo y qué ocurre en ella (Coll et.al., 1992).

En el trabajo de Badia, Barberà, Guasch y Espasa (2011) se analiza la interacción educativa producida en foros de conversación asincrónica, con el propósito de determinar patterns of educational interaction. De modo similar, en nuestra propuesta, el estudio particular de los episodios de interacción educativa persigue descubrir estas secuencias prototípicas que caracterizan un tipo de práctica con uso de TIC, con la salvedad que en nuestro caso se analizan entornos presenciales.

Por otra parte Jeong (2005), advierte que no hay consensos claros respecto de la investigación sobre la interacción educativa, particularmente en relación con teorías, métodos y herramientas que se requieren para la comunicación mediada por TIC. En este trabajo se presenta una metodología de investigación en siete etapas. De esta forma emerge un modo de hacer investigación educativa para comprender los usos de TIC a través de la configuración de patrones entre práctica pedagógica y concepciones

Usos educativo de las TIC y su relación con las concepciones del profesor de historia sobre aprender y enseñar 
educativas (Inan, Lowther, Ross y Strahl 2010; Tigchelaar, Vermunt y Brouwer, 2012).

Nuestra perspectiva asume un posicionamiento teórico constructivista respecto de las interacciones educativas que ocurren en un aula, analizándolas desde esta óptica a través de las distinciones entre interactividad pedagógica entre actores y con la tecnología, por un lado, y entre uso ideal y real de las TIC, por otro. Pues, concebimos que la manera de entender y evaluar la calidad de los procesos de enseñanza y aprendizaje mediados por TIC supone comprender no sólo los resultados en el rendimiento escolar, sino más bien cómo las TIC transforman una práctica generando un particular modo de relación entre los aprendices, el profesor y el contenido.

\section{Metodología}

En esta investigación realizamos un estudio de casos de tipo múltiple, ya que busca la comprensión del fenómeno global a partir del estudio profundo de varias situaciones (Stake, 1999).

Asumimos un tipo de muestreo intencionado (Baray, 2006), para obtener profesores "representativos" y "diversos", un criterio en la selección de los informantes fue contemplar la presencia de tres profesores en cada una de los niveles categoriales de concepciones sobre aprender y enseñar con TIC construidos teóricamente, para lo cual se aplica una entrevista semiestructurada ad hoc.

Con el propósito de interrelacionar las concepciones sobre aprender y enseñar con uso de las TIC con las prácticas docentes que ofrecen los profesores de historia a sus estudiantes, el estudio contempla la caracterización de dichas concepciones y de la práctica educativa de los diez profesores, a través de un análisis de orden interpretativo. En este sentido, el análisis de orden interpretativo cumple con relacionar los resultados empíricos obtenidos, respecto de lo que el profesor hace con las TIC con sus concepciones sobre aprender y enseñar historia, estableciendo a través de reflexiones juiciosas dichas conexiones, "la reflexividad supone dirigir la mirada hacia la persona que investiga, el reconocimiento de los supuestos teóricos y también personales" (Sandin, 2003:126).

Para efectos de la investigación, los patrones de uso de TIC, corresponden a

Usos educativo de las TIC y su relación con las concepciones del profesor de historia sobre aprender y enseñar 
pautas o modelos de comportamientos, que manifiesten modos de usar la TIC según la concepción preferente de los profesores, agrupados en sus concepciones preferentes identificadas en las entrevistas. En este estudio se establecen relaciones en torno a los tipos de uso de las TIC en 10 secuencias didácticas (en adelante SD) con las concepciones sobre aprender y enseñar historia con TIC de los 10 profesores que ejecutaron dichas experiencias pedagógicas.

Una secuencia didáctica (SD), corresponde a la macro unidad de análisis de la práctica docente con uso de TIC, en este caso cada profesor desarrolla una SD que ha sido por él propuesta. La característica principal de una SD según Coll, Colomina, Onrubia y Rochera. (1992) es que configura un proceso de enseñanza y aprendizaje completo, es decir implica en una micro escala todos los componentes de éste proceso, las etapas de inicio, desarrollo y cierre, el uso de materiales y recursos hasta concluir su uso, diferentes actuaciones de profesores y estudiantes, actividades evaluativas, entre otras. Cada SD, se analiza en función de dos niveles:

1) Segmentos de Actuación Docente (SAD). A partir de las filmaciones de las sesiones de cada profesor se construye una tipología que define tipos de usos educativos de la TIC, en función de lo que hacen los profesores y de las actividades que propone a los estudiantes. Este análisis de los videos se realiza por medio de los $\mathrm{SAD}$ que se definen como estructuras homogéneas de acción del docente o los estudiantes respecto del uso (o no uso) que le dan a las TIC durante una $\mathrm{SD}$. De esta forma, los $\mathrm{SAD}$, pueden ser aquellos en que preferentemente las TIC son utilizadas por el profesor (SADP) o cuando es el estudiante quien las utiliza (SADE).

2) Episodios de Interacción Educativa con TIC (EI-TIC), corresponden a segmentos que permiten describir el modo o forma de relación que se muestra en los videos respecto de cómo el profesor interactúa o se relaciona con los alumnos/as y con las TIC. En este sentido y a diferencia de los SAD los episodios corresponden a modos específicos de interacción entre estudiantes, profesores y TIC en las sesiones y que obedecen más bien al despliegue emergente de la propuesta de enseñanza.

\section{Resultados}

Usos educativo de las TIC y su relación con las concepciones del profesor de historia sobre aprender y enseñar 
Para establecer la presencia de patrones en el uso de TIC relacionado a una concepción en particular, asociaremos a los profesores según concepciones preferentes, es decir un primer grupo constituido por P2, P4, P5 y P10 (GP1), un segundo grupo conformado por P1, P3 y P7 (GP2), un tercer grupo que incluye a P6, P8 y P9 (GP3). Las relaciones se realizan en función de semejanzas y diferencias según el análisis fenomenográfico de las entrevistas y su organización en las tres categorías construidas teóricamente.

Gráfica 1. Concepciones preferentes de los profesores sobre aprender y enseñar

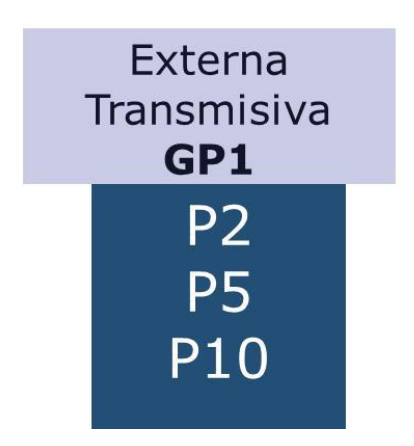
Historia con TIC

Por su parte, las diez Secuencias Didácticas (SD) dan cuenta de una homogeneidad de usos de parte de profesores y estudiantes (Arancibia y Badia, 2013). Los cuatro Segmentos de Actuación Docente (SAD) en que preferentemente el profesor usa las TIC (SADP) se concentran básicamente en modos de pasar contenidos o modelar productos, en otras palabras, se usa un recurso previamente creado ya sea por el profesor (PowerPoint), por sus estudiantes (blog) o agentes externos (video). En tanto, los $7 \mathrm{SAD}$ en que los estudiantes usan las TIC (SADE) se caracterizan por ser tipos de uso básicamente referidos a navegación en la Web o a en recursos para apoyar exposiciones, con una baja presencia, por un lado, de usos creativos orientados a la publicación o construcción de ambientes digitales (Sigalès et al., 2009) y, por otro lado, de usos comunicativos encaminados a la publicación y discusión de los contenidos (Cobo y Moravec, 2011).

La interrelación entre los tipos de uso educativo de TIC en estos 10 profesores con los grupos de profesores que distinguimos según concepción preferente sobre aprender y enseñar con TIC permitió establecer con claridad los siguientes patrones prototípicos que se resumen en el cuadro 2 y se detallan a continuación. 
Cuadro 2. Patrones de uso según concepciones sobre aprender y enseñar historia con TIC

\begin{tabular}{|l|c|c|c|}
\hline \multicolumn{1}{|c|}{ Tipo de Uso e interacción con TIC en la Secuencia Didáctica } & GP1 & GP2 & GP3 \\
\hline Apoyo a la exposición oral del profesor & $30 \%$ & $4 \%$ & $6,7 \%$ \\
\hline Uso de TIC estudiantes & $67 \%$ & $85 \%$ & $92,5 \%$ \\
\hline Estudiantes elaboran productos de aprendizaje con TIC & $9,2 \%$ & $21,2 \%$ & $48 \%$ \\
\hline Profesor expone contenido con apoyo de Presentación & $22,2 \%$ & $1,6 \%$ & $1,0 \%$ \\
\hline El profesor apoya el desarrollo de la tarea por grupos de estudiantes & $16,5 \%$ & $62,2 \%$ & $26,4 \%$ \\
\hline El profesor observa a toda el aula el desarrollo de la tarea & $9,6 \%$ & $6,0 \%$ & $28,4 \%$ \\
\hline El profesor resuelve dudas sobre el contenido por grupos & $21 \%$ & $1,3 \%$ & $6,0 \%$ \\
\hline
\end{tabular}

Fuente: propia

\section{a) GP1.}

El GP1 presenta, una alta coincidencia en términos que son profesores que más usan las TIC ellos directamente (25\% del tiempo de las SD) y por ende, son las SD en que, porcentualmente, menos las usan los estudiantes (75\% de uso).

Exhiben una nítida semejanza en cuanto a que son los profesores que mayormente usan las TIC como apoyo a la exposición para presentar contenidos, otorgándole en ambos casos cerca del tercio de sus usos a dicha utilización. Además lo hacen interactuando de modo muy similar.

En cuanto a los EI-TIC, fue posible encontrar asociaciones entre estos profesores, tal cual se mostró en la tabla II.2.27, en los EI-TIC 8, 15 y 21. Es decir, una concepción de orden externa transmisiva favorecería el trabajo con TIC como soporte para el docente para exponer contenido con apoyo de presentación, asimismo hay una tendencia a supervisar el trabajo de los estudiantes a través de la realización de preguntas orales a toda el aula para controlar la comprensión y la resolución de las dudas son preferentemente sobre el contenido.

En otro sentido, en cuanto al resto de los SAD y EI-TIC no fue posible encontrar alguna asociación. Por ejemplo, como se ha demostrado en la tabla II.2.20.1, referida a los SADE, entre los profesores que manifiestan similares concepciones sobre aprender y enseñar historia con TIC no se encuentran asociaciones en cuanto a los usos que les dan sus estudiantes a las TIC.

Como se ha podido constatar, existe un patrón común entre las secuencias 
didácticas del GP1, más bien, en orden a los SAD en que las TIC son usadas por el profesor, así como también, en cuanto a los EI-TIC el vínculo entre estos profesores se encuentra en la D2, relacionado con los formatos para exponer contenidos.

\section{b) GP2.}

A nivel de $\mathrm{SAD}$, se puede indicar que para estos profesores, los estudiantes son los que porcentualmente más tiempo utilizan las TIC (85\%), tal como se expuso en el cuadro 2.

En cuanto a los EI-TIC, del GP2 fue posible encontrar vinculación en que no son las SD en que menos se usan las TIC para exponer contenido con apoyo de presentación (EI-TIC8), asimismo son los profesores que más les preocupa apoyar el desarrollo de la tarea por grupos (EI-TIC15), así como también son lo que menos muestran interés en resolver dudas sobre el contenido por cada grupo (EI-TIC21).

d) Cabe señalar que P1 y P7 cuando usan la TIC ellos (SADP) lo hacen de modo preferente para apoyar la conversación con los estudiantes en tanto P3 lo hace para motivarlos con la exposición de un material audiovisual.

Este GP2 exhibe una asociación en la presencia del EI-TIC15, es decir son profesores muy preocupados de apoyar las tareas por cada grupo. Al contrario, P1 y P3 no presentan episodios en que expongan contenidos oralmente con apoyo de presentaciones (EI-TIC8), ni tampoco se les observa preocupados por resolver dudas sobre el contenido (EI-TIC21). Existe, por tanto un patrón vinculado a los EI-TIC relacionados al apoyo del trabajo de los estudiantes, el cual se hace preferentemente en función de la tarea y de modo grupal.

Igualmente, el GP2 no manifiesta similares SAD en cuanto a despliegue, según mostraron los análisis de los respectivos diagramas, en sus propuestas de uso de TIC dentro de sus SD.

En este sentido, estos profesores manifiestan en SAD en que los usos de TIC son acordes con modos de uso dentro de una concepción constructivista/social. Prima, en esta concordancia las relaciones entre estos profesores en la M1, es decir respecto del curriculum de historia, pues como se ha mostrado sus concepciones están fuertemente influenciadas por un currículo crítico por tanto con presencia 
principalmente de SADE, más que de SADP, es más cuando aparecen SADP, estos son preferentemente para motivar (P3) o generar conversaciones (P1 y P7).

\section{c) GP3.}

En relación a las relaciones entre las concepciones sobre aprender y enseñar historia con TIC y las prácticas educativas del GP3 es posible identificar algunos patrones comunes de despliegue en torno principalmente a los SAD no así en función de los EI-TIC. Recordemos que estos profesores se asocian en sus concepciones en las tres metacategorías.

El principal nivel de asociación en GP3 se da a nivel de $\mathrm{SAD}$, al respecto se puede indicar que estos profesores presentan porcentualmente bastante tiempo en que las TIC las utilizan los estudiantes para elaborar productos de aprendizaje.

En cuanto a los EI-TIC, del GP3 no fue posible encontrar vinculación directa entre las 3 SD. Más bien lo que hay son prácticas diferentes, por tanto uso de TIC distintos.

Cabe señalar, que el GP3 cuando usan las TIC (SADP) lo hacen de modo similar, así los tres profesores los hacen solo para apoyar su exposición oral, aunque como hemos visto P6 lo hace muy marginalmente.

En cuanto a las diferencias, entre este GP3 observamos que si bien a los tres profesores les preocupa que sus estudiantes elaboren productos de aprendizaje a través de la búsqueda de información desde Internet, tanto P8 como P9 exhiben SAD en que los estudiantes exponen estos productos al final de la SD, lo que no ocurre con P6.

Igual que los grupos anteriores, el GP3 no manifiesta similares SAD en cuanto a despliegue, según mostraron los análisis de los concernientes diagramas, en sus propuestas de uso de TIC dentro de sus SD.

\section{Discusión de los resultados presentados}

Establecidos algunos de los patrones de asociación entre concepciones preferentes sobre aprender y enseñar historia con TIC y los usos educativos que ofrece el profesor a sus estudiantes, podemos aseverar que los profesores que presentan concepciones similares tienden a organizar desde un punto de vista estructural su secuencia didáctica de modo parecido en términos de SAD en que el profesor las usa y en los EI-TIC. Sin embargo, este vínculo no es tan nítido en los SAD en que las usan los estudiantes 
(SADE), así como en el despliegue temporal y secuencial de dichos segmentos durante el despliegue de las SD.

Un ejemplo de lo dicho, es que dos profesores que se han clasificado en una concepción de tipo externa transmisiva (P2 y P5), tienden a tener muchos minutos en los $\mathrm{SAD}$ con exposiciones de contenido disciplinar, pero estos episodios de exposición por un lado se distribuyen de diferente forma en el tiempo de la SD y además se realizan de diferente manera, con distintos énfasis o recursos (power point, videos, documentos, seguidos en telón, en pantalla, entre otros), así como entre los SADE de sus SD no hay mayores coincidencias. Sin embargo en los EI-TIC se vuelven a encontrar vínculos entre ellos tanto en el modo de supervisar el trabajo como en las interacciones para exponer contenidos.

Según lo expuesto, las concepciones sobre aprender y enseñar historia con TIC identificadas y categorizadas en los profesores, guardan un nivel de vinculación con los usos educativos de las TIC identificados en las respectivas SD, esta evidencia es consistente con los estudios de Boulton-Lewis et.al. (2001), Both (1997), Drenoyanni y Selwood (1998), Matzen y Edmunds (2007), entre otros.

El tipo de relación que es posible establecer se da en función de la estructuración de los segmentos de actuación (SAD), o sea, de los grandes bloques didácticos que aparecen respecto de los tipos de uso de las TIC. Vale decir las relaciones entre concepciones y práctica se conectan en las actividades definidas y su intencionalidad, no así en el despliegue mismo de la SD ni en el modo concreto en que los profesores interactúan con sus estudiantes.

Según lo establecido, estos resultados ubican la investigación en una posición intermedia entre aquellos estudios que plantean que sí existe incidencia de las creencias sobre las prácticas (Hermans et al., 2008; Matzen y Edmunds, 2007) y entre aquellos que como Chen (2008) dicen que no existe tal influencia. En tal sentido, si bien consta un nivel de incidencia de las concepciones sobre el uso educativo de las TIC, esta influencia no viene a condicionar tipos de uso estandarizados según concepción preferente, más bien el nivel de ascendencia es en algunos aspectos, como ya se ha dejado en claro, principalmente en torno al uso que le dan los estudiantes en cuanto a mayor tiempo y al para qué las usa el profesor.

Usos educativo de las TIC y su relación con las concepciones del profesor de historia sobre aprender y enseñar 
Por tanto que las concepciones condicionen la práctica con TIC de los profesores de historia de la muestra tiene real significancia en función de comprender que las prácticas escolares, las que en definitiva dificultan o permiten procesos de aprendizaje profundo en los estudiantes, vienen mediadas por el pensamiento del profesor respecto de cuestiones de orden esencialmente técnico-pedagógico, el currículum y la didáctica.

En la actualidad los procesos de cambio en la escuela y en las prácticas escolares se asocia de modo irreductible al uso educativo de las TIC en los contextos escolares (Meier, 2005; LLoyd y McRobbie, 2005), con lo cual parece que no es posible en el presente instalar la innovación didáctica sin uso de TIC. Sin embargo, como hemos demostrado los usos tradicionales están aún vigentes en cuanto a incorporación de TIC, permeando incluso prácticas de profesores cuya concepción es de tipo constructivista. Aparentemente, la utilización de estos recursos es subsumida por la tradición en un contexto escolar principalmente reproductor y rutinario, de allí que sea necesario insistir en la relevancia de estudiar en qué medida las prácticas pedagógicas están condicionadas por las concepciones de los profesores y su relación con otras variables de contexto (Ruthven et al., 2004).

\section{Conclusiones}

En primer lugar, se ha demostrado que las relaciones entre concepciones y las prácticas con uso de TIC se dan a ciertos niveles dentro de las respectivas secuencias didácticas. Estas influencias se presentan en todos los profesores del estudio. Es decir, existe incidencia de las concepciones sobre aprender y enseñar historia sobre los usos educativos de las TIC en profesores de concepciones externa/transmisiva y constructivistas.

Una segunda conclusión establece que el tipo de concepciones sobre enseñar y aprender que tenga el profesor, influye en cómo se reparte el tiempo de uso de las TIC entre el propio profesor y sus alumnos. Los profesores de la muestra con una concepción externa/transmisiva (P2, P5 y P10), tienden a usar más tiempo el ordenador, mientras que los profesores con una concepción de orden constructivista (P1, P3, P6, P7, P8 y P9) exhiben las SD con mayor uso de TIC de parte de los estudiantes. 
Una tercera conclusión indica que hay patrones de usos prototípicos de las TIC asociados a concepciones preferentes. Esta relación está referida a que profesores de similares concepciones desarrollan su docencia mediante usos de TIC parecidos. Por tanto, existen ciertos patrones que es posible identificar en el uso de TIC en clases de historia, asociados a un tipo de concepción preferente sobre aprender y enseñar.

La principal conclusión a la que llega esta investigación es que existe una interrelación posible de establecer entre concepciones sobre enseñar y aprender y prácticas docentes con TIC. Esta conexión entre concepciones y uso de TIC se evidencia en los patrones de usos prototípicos establecidos por grupos de profesores según concepción preferente (GP1, GP2 y GP3). Ello nos hace suponer, que los usos educativos de las TIC están integrados dentro del sistema didáctico de un profesor como un elemento en alguna medida supeditado a sus concepciones sobre Enseñar y Aprender. Estas concepciones operan como un conocimiento regulador que prevalece y es utilizado como uno de los criterios que considera el profesor al momento de seleccionar qué tipos de TIC son asumibles y, por lo tanto coherentes, con un tipo de concepción, y también cómo serán usadas estas TIC para poner en juego una determinada intencionalidad educativa en las aulas.

Respecto de esto, la toma de decisiones didácticas que efectúa un profesor está influenciadas por sus concepciones, de allí que emerja como fundamental el conocimiento explícito de estas concepciones si lo que se pretende es cambiar una práctica pedagógica. El alcance de este planteamiento se extiende más allá del uso de TIC en contextos escolares, expresándose como aspecto fundamental del trabajo didáctico en la formación de profesores de historia.

En consecuencia, un conjunto de concepciones sobre el curriculum de historia y sobre la acción didáctica que conviven dentro del sistema cognitivo de un profesor guían u orientan de modo parcial los usos de TIC, su incidencia se da principalmente respecto al para qué la usa el profesor y de algunos tipos de interacción. Con ello consideramos que además es necesario indagar en la presencia de otros factores de tipo contextuales, que junto a las concepciones terminan configurando las decisiones y acciones que toma el docente, por tanto que también presentan un nivel de incidencia, tal cual los indagan los trabajos de García-Valcárcel y Tejedor (2010) y De Pablos, 
Colás y González (2010) o en términos específicos los aspectos culturales y las actitudes ante las TIC, que pueden potenciar o inhibir su uso en el aula (Albirini, 2006).

En efecto, los resultados no nos permiten aseverar con absoluta certeza que las concepciones definen o condicionan las prácticas pedagógicas, más bien, lo que sí es posible conjeturar es una idea a modo de hipótesis en torno a que la relación está definida por la presencia de un conjunto de concepciones sobre enseñar y aprender historia, las que determinan la presencia de un conjunto de prácticas con TIC. Lo que proponemos luego de realizado este estudio, es que se ha de contemplar esta idea en futuros estudios que trabajen sobre las variables, condiciones o aspectos que inciden sobre las prácticas pedagógicas o en la toma de decisiones didácticas.

\section{Referencias bibliográficas}

Albirini, A. (2006). Teachers' attitudes towards information and communication technologies: The case of Syrian EFL teachers. Computers \& Education, 47, 373-398.

Arancibia, M. y Badia, A. (2013). Caracterización y valoración de los usos educativos de las TIC en 10 secuencias didácticas de historia en enseñanza secundaria. Estudios Pedagógicos 39, No. Especial (7-24)

Arancibia, M.; Soto, C.P.; Contreras, P. (2010). Concepciones del profesor sobre el uso educativo de las TIC asociada a procesos de enseñanza-aprendizaje en el aula escolar. Estudios Pedagógicos 36, V.1. (23-51)

Arancibia, M. (2011). Tradiciones psicoeducativas y concepciones sobre aprender y enseñar con TIC (87-142). En: Hernández, R.; Padilla, S. y Arancibia, M. (eds). Educación Superior, Innovación e Internacionalización. Ensayos, proyectos y experiencias. Edic. Univ. De Guadalajara: Guadalajara

Badia, A., Barberà, E., Guasch, T., Espasa, A. (2011). Technology educational affordance: Bridging the gap between patterns of interaction and technology usage. In: Digital Education Review, 19, 20-35. doi:http://greav.ub.edu/der $[30 / 11 / 2011]$. 
Barba, C. y Capella, S. (coords.) (2010). Ordenadores en el aula. La clave es la metodología. Barcelona: Grao.

Boulton-Lewis, G., Smith, D., McCrindle, A., Burnett, P. y Campbell, K. (2001). Secondary teachers' conceptions of teaching and learning. Learning and Instruction 11, 35- 51.

Chen, C. (2008). Why Do Teachers Not Practice What They Believe Regarding Technology Integration?. The Journal of Educational Research. 102(1). 65-75.

Coll, C., Colomina, R., Onrubia, J. y Rochera, M (1992). Actividad conjunta y habla: una aproximación al estudio de los mecanismos de influencia educativa. Infancia y aprendizaje, 59-60, 189-232.

Coll, C. Mauri, T. y Onrubia, J. (2008). El análisis de los procesos de enseñanza y aprendizaje mediados por las TIC: una perspectiva constructivista $(47-62)$. En: Barberá, E., Mauri, T. y Onrubia, J. (2008). Cómo valorar la calidad de la enseñanza basada en las TIC. Barcelona: GRAO

Colomina, R., Mayordomo, R. y Onrubia, J. (2001). El análisis de la actividad discursiva en la interacción educativa. Algunas opciones teóricas y metodológicas. Infancia y aprendizaje, 93, 67-80.

Drenoyianni, H. y Selwood, I. (1998). Conception or misconceptions? Primary teachers'perceptions and use of computers in the classroom. Education and information Technologies 3, 87-99.

ENLACES (2010). Centro de Educación y Tecnología del Ministerio de Educación. El Libro Abierto de la Informática Educativa. Lecciones y Desafíos de la Red Enlaces (2010). Santiago: Publicación de Enlaces.

Gómez, V.; Guerra, P. (2010). Teorías implícitas respecto a la enseñanza y el aprendizaje: ¿Existen diferencias entre profesores en ejercicio y estudiantes de pedagogía?. Estudios Pedagógicos XXXVIII, Nº 1. 25-43.

Gutiérrez, A. y Tyner, K. (2012). Educación para los medios, alfabetización mediática y competencia digital. Comunicar 38, v. XIX; $31-39$.

Hermans, R., Tondeur, J., Van Braak, J. y Valcke, M. (2008). The impact of primary

Usos educativo de las TIC y su relación con las concepciones del profesor de historia sobre aprender y enseñar 
school teachers' educational beliefs on the classroom use of computers. Computers \& Education 51. 1499-1509.

Inan, F., Lowther, D., Ross, S. y Strahl, D. (2010). Pattern of classroom activities during students' use of computers: Relations between instructional strategies and computer applications. Teaching and Teacher Education 26. 540-546.

Jeong, A. (2005). A Guide to Analyzing Message-Response Sequences and Group Interaction Patterns in Computer mediated Communication. Distance Education. $26(3), 367-383$.

Kim, C., Kim, M., Lee C., Spector M. y DeMeester, K. (2013). Teacher beliefs and technology integration. Teaching and Teacher Education, 29, 76 - 85

Lin, H. y Tsai, Ch. (2011). College students' conceptions of learning management: the difference between traditional (face-to-face) instruction and Web-based learning environments. Learning, Media and Technology, 36:4, 437-452

Levin, T. y Wadmany, R. (2006). Teachers' Beliefs and Practices in Technology-based Classrooms: A Developmental View. Journal of Research on Technology in Education, 39(2), 157-181.

Matzen, N. y Edmunds J. (2007). Technology as a Catalyst for Change: The Role of Professional Development. Journal of Research on Technology in Education, 39(4). 417-430.

Phelps, R., Graham, A. y Watts, T. (2011). Acknowledging The Complexity And Diversity Of Historical And Cultural ICT Professional Learning Practices In Schools. Asia-Pacific Journal Of Teacher Education, 39(1), 47-63.

Prestridge, S. (2012). The beliefs behind the teacher that influences their ICT practices. Computers \& Education, 58 449-458.

Ricoy, Mª C., Feliz, T. y Sevillano Ma L. (2010). Competencias para la utilización de las herramientas digitales en la sociedad de la información. EducaciónXX1, 13.1, 199-219.

Ruthven, K., Hennessy, S. y Brindley, S. (2004). Teacher representations of the successful use of computer-based tools and resources in secondary-school

Usos educativo de las TIC y su relación con las concepciones del profesor de historia sobre aprender y enseñar 
English, Mathematics and Science. Teaching \&Teacher Education, 20 (3), 259275.

Sigalès, C., Mominó J., Meneses, J. y Badia, A. (2009). La integración de internet en la educación escolar española. Barcelona: Ariel.

Tigchelaar, A.; Vermunt, J. y Brouwer, N. (2012). Patterns of development in secondcareer teachers' conceptions of learning and teaching. Teaching and Teacher Education, 28, 1163 - 1174

Usos educativo de las TIC y su relación con las concepciones del profesor de historia sobre aprender y enseñar 